\title{
Miltefosine shows fungicidal effect on Cryptococcus spp. by apoptosis mechanism.
}

\author{
SPADARI C.C. ${ }^{1}$; VILA T. ${ }^{2}$; ROZENTAL S. ${ }^{3}$, ISHIDA K. ${ }^{1}$
}

ICBUSP

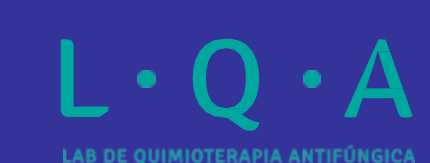

INTRODUCTION

Cryptococcus neoformans and Cryptococcus gattii are the etiological agents of cryptococcosis, a disease with a high incidence among severely immunocompromised patients. Amphotericin B, fluconazole, and flucytosine combined with amphotericin B are the alternatives of treatment for severe cryptococcal infection, but its use has been limited. Thus, the search for new therapeutic options is necessary for the treatment of this mycosis. Previous studies had shown miltefosine (MFS) as an alternative antifungal drug for several pathogenic fungi including Cryptococcus spp; ; however, its mechanism of action on this fungus is poorly understood. The aim of this study was to evaluate the antifungal effects of MFS on Cryptococcus neoformans (H99 and CAP59) and Cryptococcus gattii (ATCC 56990).

\section{RESULTS}

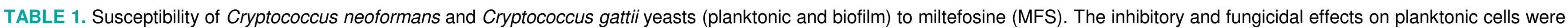

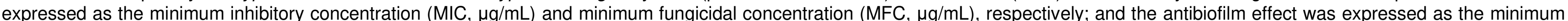

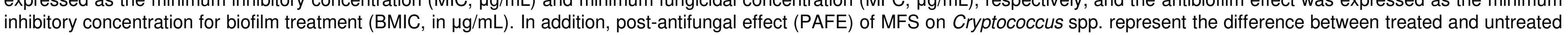

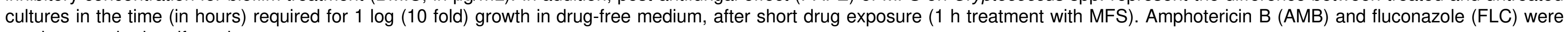
used as standard antifungals.

\begin{tabular}{|c|c|c|c|c|c|c|c|c|c|c|}
\hline \multirow[t]{2}{*}{ Strains } & \multicolumn{2}{|c|}{ AMB } & \multicolumn{2}{|c|}{ FLC } & \multicolumn{6}{|c|}{ MFS } \\
\hline & MIC & MFC & $\mathrm{MIC}$ & MFC & $\mathrm{MIC}$ & MFC & $\mathrm{B}_{\mathrm{S}} \mathrm{MIC}$ & $\mathrm{B}_{\mathrm{D}} \mathrm{MIC}$ & PAFE 1x MIC & PAFE 4x MIC \\
\hline C. neoformans CAP59 & 0.03 & 0.03 & 1 & 32 & 1 & 1 & 8 & 0.5 & 8.15 & * \\
\hline C. neoformans H99 & 0.25 & 0.25 & 4 & 4 & 2 & 2 & $>16$ & 2 & 2.71 & 6.73 \\
\hline C. gattii ATCC 56990 & 0.25 & 0.25 & 2 & 32 & 0.5 & 0.5 & 16 & 1 & 0 & 2.75 \\
\hline
\end{tabular}

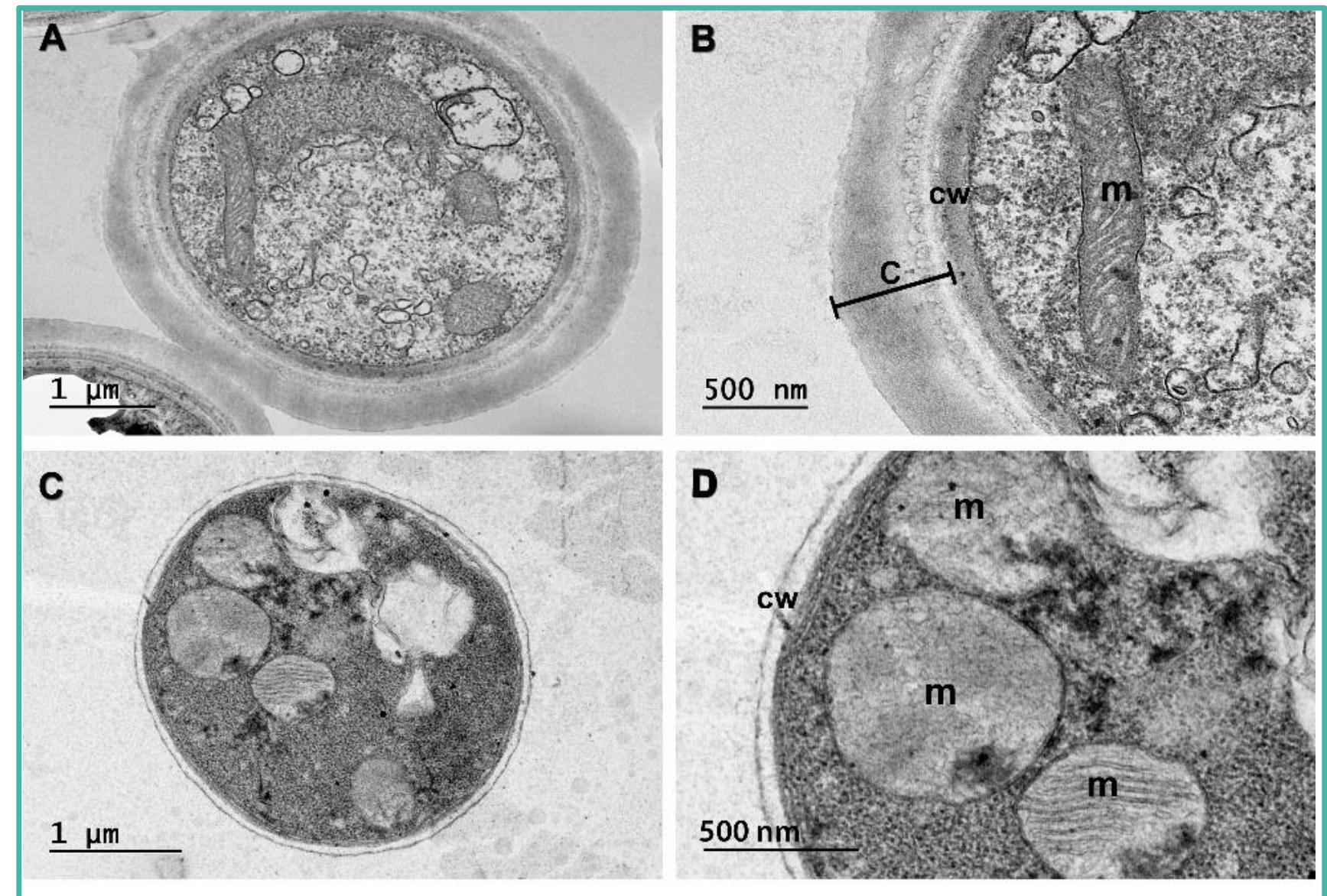

FIGURE 1. Transmission electron microscopy images of Cryptococcus neoformans H99 yeast. A, B - untreated yeasts; C, D -yeasts treated with $1 \mu \mathrm{g} / \mathrm{mL}$ MFS, showing a loss of the mucopolysaccharide capsule, as well as alterations in the mitochondrial ridges, and mitochondrial swelling. respectively. $\mathbf{m}$, mitochondria; $\mathbf{c w}$, cell wall; $\mathbf{c}$ capsule.

TABLE 2 Effect of exogenous ergosterol on the minimum inhibitory concentration (MIC, in $\mu \mathrm{g} / \mathrm{mL}$ ) of miltefosine (MFS) and amphotericin B (AMB) on Cryptococcus neoformans (H99 reference strain and CAP59 acapsular strain) and C. gattii (ATCC 56990 reference strain).

\begin{tabular}{ccccccc}
\hline & \multicolumn{2}{c}{$\begin{array}{c}\text { C. neoformans } \\
\text { CAP59 }\end{array}$} & \multicolumn{2}{c}{$\begin{array}{c}\text { C. neoformans } \\
\text { H99 }\end{array}$} & \multicolumn{2}{c}{$\begin{array}{c}\text { C. gattii ATCC } \\
56990\end{array}$} \\
\hline $\begin{array}{c}\text { Ergosterol } \\
(\mu \mathrm{g} / \mathrm{mL})\end{array}$ & MFS & AMB & MFS & AMB & MFS & AMB \\
0 & 1 & 0.03 & 1 & 0.12 & 0.5 & 0.25 \\
50 & 4 & 0.12 & 8 & 0.5 & 2 & 0.5 \\
100 & 8 & 8 & 16 & 1 & 8 & 1 \\
200 & 16 & 16 & 16 & 2 & 8 & 8 \\
\hline
\end{tabular}

$\square$ Extravasation of DNA and proteins through the membrane in significant amount were also observed when yeasts were treated with $2 x$ and $4 x$ MIC of MFS.

FIGURE 3. Model of the mechanism of action of miltefosine (MFS) on Cryptococcus spp. yeasts. MFS interacts with cell membranes leading to fungal death by the following important mechanisms: (i) MFS acts as a detergent, interacting with phospholipids and/or ergosterol, which alters the plasma membrane permeability; (ii) Once inside the cell, MFS acts on the mitochondria, leading to mitochondrial swelling and loss of membrane potential, and promoting ROS. Then, DNA condensation and fragmentation lead to yeast cell death by apoptosis. Another effect observed in Cryptococcus spp. yeasts was a reduction in the mucopolysaccharide capsule.
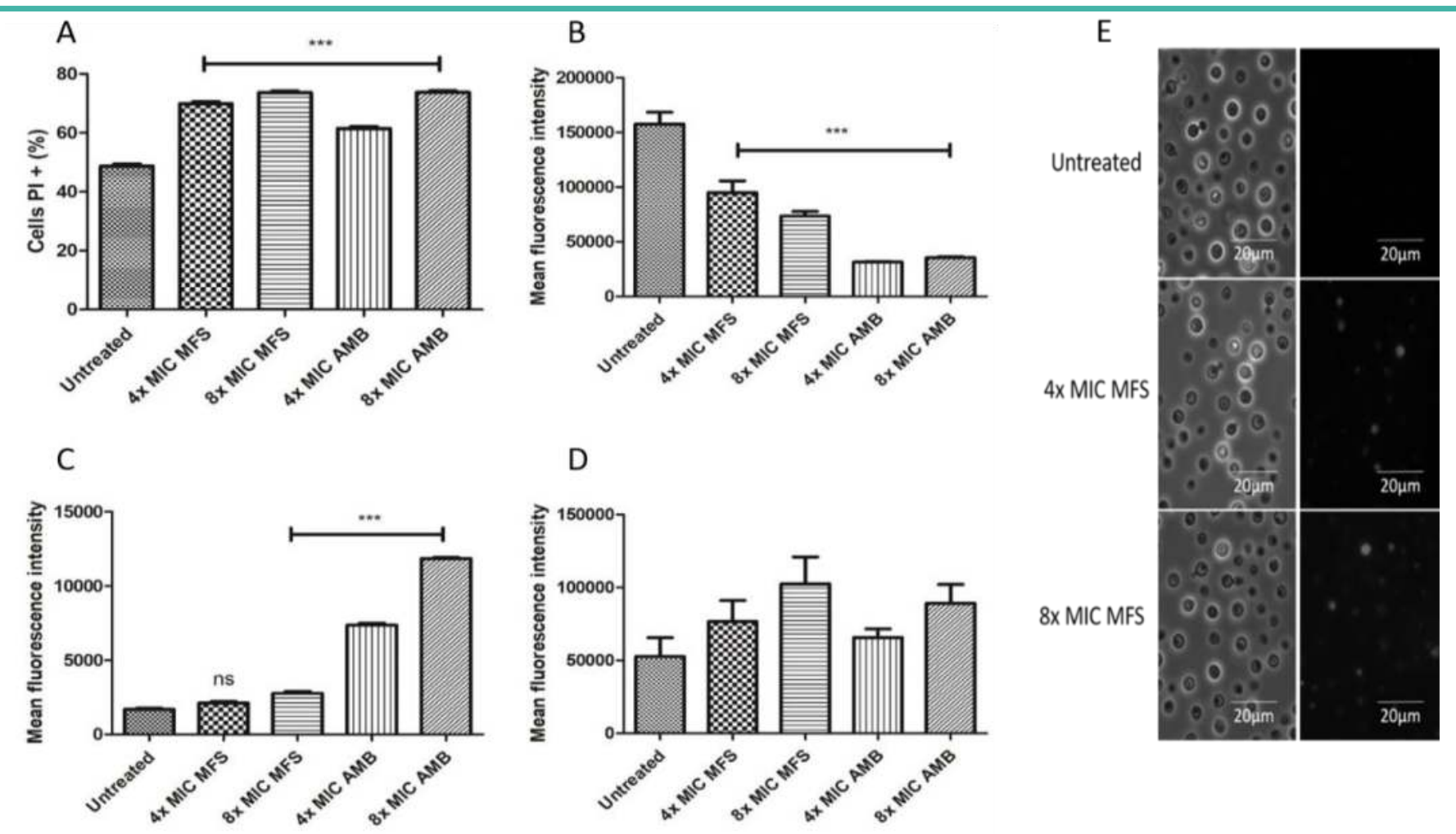

FIGURE 2. Yeasts of Cryptococcus neoformans $\mathrm{H} 99$ untreated or treated with fungicidal concentrations of miltefosine (MFS) or amphotericin B (AMB) (4x and $8 \times$ MIC for $24 \mathrm{~h}$, at $35^{\circ} \mathrm{C}$ were analyzed by flow cytometry or fluorescence microscopy. (A) Percentage of yeasts labeled with propidium iodate $(\mathrm{PI})$; (B) Mean fluorescence intensity of yeasts labeled with Rhodamine123, (C) 2'7'-Dichlorofluorescin diacetate (DCFH-DA) and, (D) terminal deoxynucleotide transferase dUTP nick end labeling (TUNEL). (E) The chromatin condensation (white spots) in treated yeasts with 4x and 8x MIC of MFS was observed by fluorescence microscopy (right column) after DNA staining with Hoechst 33342 whilst untreated yeasts presented absence of marking. Phase contrast images of the same fields are shown (E, left column). ${ }^{* * *} \mathrm{P}<0.05$ compared with untreated yeasts (one-way ANOVA with Tukey's posttest).

whe Tuk's postles.

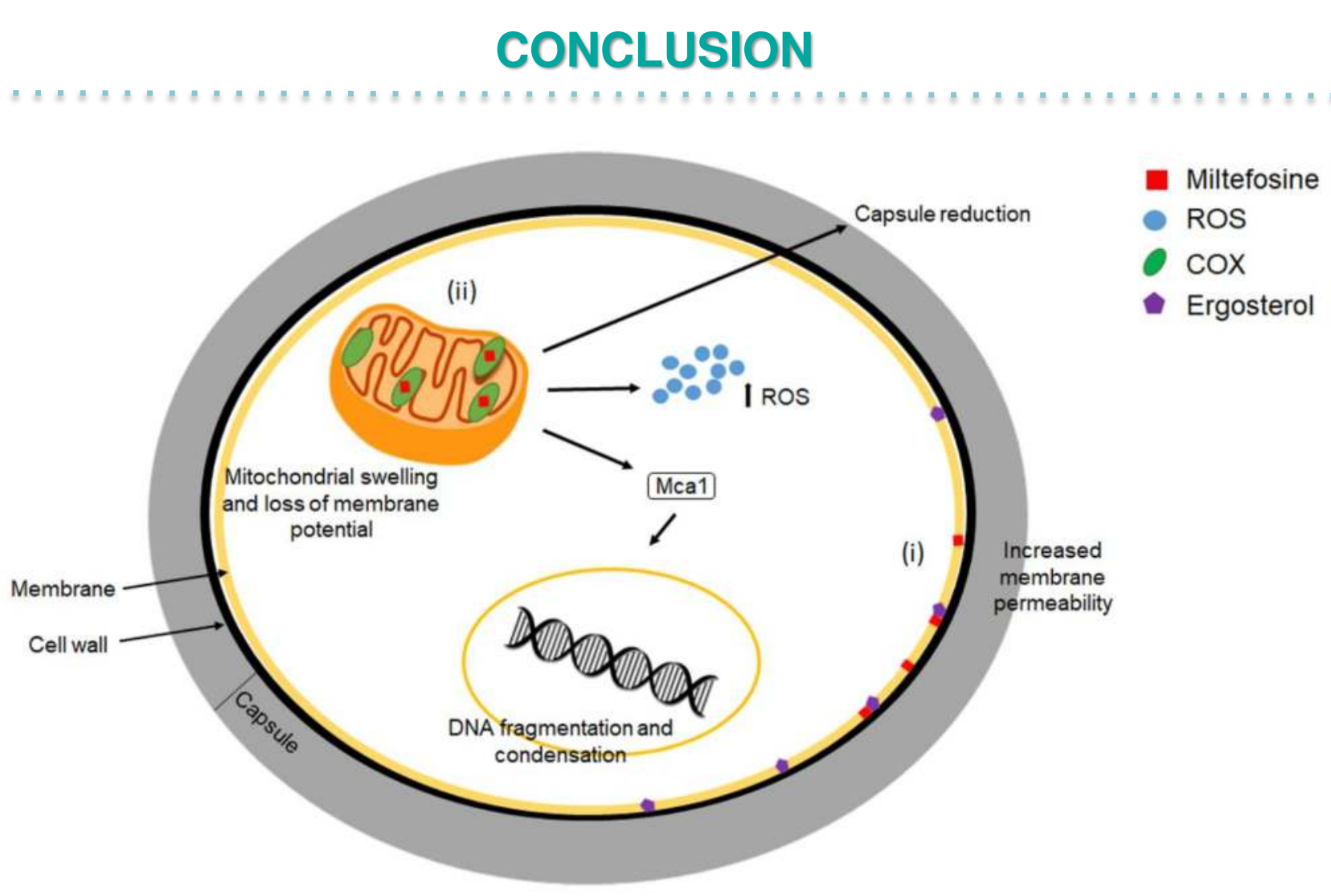

